\title{
Análisis documental: importancia de los entornos virtuales en los procesos educativos en el nivel superior
}

\author{
Luisa Rosa Isela Aguilar Vargas
}

Estudiante de la maestría en Tecnología Educativa de la Universidad Da Vinci y profesora de la Facultad de Educación de la Universidad Autónoma de Yucatán laguilar19@udavinci.edu.mx

\section{Emma Omolade Otuyemi Rondero}

Estudiante de la maestría en Tecnología Educativa de la Universidad Da Vinci y licenciada en Gastronomía por la Universidad Popular Autónoma del Estado de Puebla eotuyemi19@udavinci.edu.mx

\section{Extracto}

El presente estudio tuvo como propósito conocer la importancia de los entornos virtuales en los procesos educativos en el nivel superior. Se llevó a cabo una revisión sistemática de la literatura de 22 trabajos científicos publicados entre 2011 y 2019 en diferentes países de América Latina y Europa. Los resultados son los siguientes: el concepto de «entornos virtuales» se asocia a un software o a una aplicación informática, espacio o medio en la red que facilita la comunicación. Sus características son la colaboración, la interactividad, la flexibilidad, la estandarización y la escalabilidad. Los espacios virtuales son utilizados para mejorar el proceso de enseñanzaaprendizaje, promover el desarrollo de habilidades interpersonales, complementar la educación presencial y facilitar el seguimiento del aprendizaje. Entre sus beneficios se encuentran la calidad educativa del aprendizaje y la motivación. Los obstáculos para su uso son el temor al cambio, el poco interés de los alumnos y la falta de infraestructura adecuada en las instituciones. En conclusión, los entornos virtuales son espacios que facilitan la comunicación a los alumnos y el acceso a diversos materiales y recursos. Principalmente, se caracterizan por su interactividad, flexibilidad, escalabilidad y ubicuidad respecto al aprendizaje. Son un mecanismo de motivación y evaluación.

Palabras clave: entornos virtuales de aprendizaje; procesos educativos; educación superior; comunicación.

Fecha de entrada: 11-03-2020 / Fecha de revisión: 14-04-2020 / Fecha de aceptación: 15-04-2020.

Cómo citar: Aguilar Vargas, L. R. I. y Otuyemi Rondero, E. O. (2020). Análisis documental: importancia de los entornos virtuales en los procesos educativos en el nivel superior. Tecnología, Ciencia y Educación, 17, 57-77. 


\title{
Documentary analysis: importance of virtual environments in educational processes at the higher level
}

\author{
Luisa Rosa Isela Aguilar Vargas \\ Emma Omolade Otuyemi Rondero
}

\section{Abstract}

The purpose of this study was to understand the importance of virtual environments in educational processes at the higher level. A systematic literature review of 22 scientific papers published from 2011 to 2019 was carried out in different countries of Latin America and Europe. The results are as follows: the concept of «virtual environment» is associated with a software or computer application, space or medium on the network that facilitates communication. Its characteristics are collaboration, interactivity, standardization, flexibility and scalability. They are used to improve the teaching-learning process, promote the development of interpersonal skills, complement face-to-face education and facilitate the monitoring of learning. Among its benefits is educational quality, learning and motivation. The obstacles to its use are fear of change, little interest from students and the lack of adequate infrastructure in institutions. In conclusion, virtual environments are spaces that facilitate communication and access to various materials and material resources for students. Mainly, it is characterized by its interactivity, flexibility, scalability and ubiquity regarding learning. They are a mechanism of motivation and evaluation.

Keywords: virtual environments of learning; educational processes; higher education; communication. 


\section{Sumario}

1. Introducción

2. Objetivos

2.1. Objetivo general

2.2. Objetivos específicos

3. Justificación

4. Metodología

5. Resultados

5.1. Definiciones de los «entornos virtuales»

5.2. Principales características de los entornos virtuales

5.3. Usos de los entornos virtuales en los procesos educativos

5.4. Beneficios del uso de los entornos virtuales en los procesos educativos

5.5. Obstáculos del uso de los entornos virtuales en los procesos educativos

6. Discusión de los resultados

7. Conclusiones

Referencias bibliográficas 


\section{Introducción}

El contexto actual se ha visto modificado por el desarrollo y el crecimiento exponencial de la tecnología en diversos ámbitos. En este sentido, es común observar la incorporación de las tecnologías de la información y la comunicación (TIC) a la vida cotidiana de las personas. Por tal motivo,

El contexto actual se ha visto modificado por el desarrollo y el crecimiento exponencial de la tecnología en diversos ámbitos no podemos considerarlas ajenas, sino que, por el contrario, es importante reconocer que facilitan diversos procesos en nuestra vida cotidiana; por ejemplo, el acceso a la información. De este modo, uno de los contextos en los que han impactado sobremanera las TIC ha sido en el terreno educativo, debido a que, como establecen Moreira-Segura, Delgadillo-Espinoza, Sánchez-Calvo y Alvarenga-Venutolo (2014), en las instituciones educativas, el uso de las tecnologías no solo ha sido considerado una moda, sino que, con el paso del tiempo, se ha visto como algo necesario para diversificar la oferta académica.

Basándonos en lo anterior, dentro de las nuevas oportunidades que ofrece la inclusión de las TIC en la educación, podemos mencionar que, desde hace algún tiempo, se ha desarrollado un concepto relevante; a saber, la «educación virtual» o «e-learning»:

[...] los cuales implican una formación educativa online que usa las posibilidades comunicativas de internet. Gracias a estas innovaciones, la educación a distancia se ha revolucionado por completo, entrando de lleno en un nuevo paradigma: los entornos virtuales de aprendizaje (Hernández y Romero, 2011, p. 96).

También, el desarrollo de los entornos virtuales de aprendizaje ha sido considerado como una opción para la educación presencial debido a que la combinación de elementos de esta modalidad y de la virtual generan un aprendizaje híbrido, conocido como «blended learning» (Hernández y Romero, 2011).

Asimismo, tomando como base lo mencionado por Vidal y Camarena (2014), las TIC han transformado la realidad educativa; es decir, ahora las instituciones académicas y de investigación deberán aprovechar las oportunidades que las TIC han generado en este ámbito, puesto que, de otra manera, si no las utilizan, corren el riesgo de quedarse rezagadas en la oferta y demanda educativas. 
En función de lo anterior, sin duda, la oferta académica ha evolucionado con la implementación de las TIC, por lo que este contexto se ha transmutado, dado que no se ofrece únicamente un servicio educativo de modo presencial, sino que es necesario presentar nuevas alternativas que permitan establecer distintas modalidades educativas; por ejemplo, aquellas vinculadas con lo virtual, cuyo objetivo consiste en superar la distancia, deshacerse de los horarios fijos y desarrollar la facilidad de administrar el tiempo de estudio, teniendo la opción de realizar otras actividades que forman parte de la vida diaria (Moreira-Segura et al., 2014).

De esta manera, actualmente, los procesos educativos virtuales están evolucionando y transformándose, por lo que en ocasiones es difícil conceptualizarlos y precisar su abordaje. Sin embargo, existen muchos autores que han dedicado gran parte de su tiempo a estudiarlos y conceptualizar algunos de los elementos que tienen en común, como ocurre, por ejemplo, con el caso de la llamada «educación a distancia» (Jaramillo, 2012).

A partir de lo expuesto, se reconoce que en los procesos de aprendizaje actuales los ambientes virtuales resultan ser el complemento perfecto para una educación presencial. Se pretende que coexistan con sus capacidades y estrategias de manera coherente, con la finalidad de adquirir diversas habilidades orientadas a lograr «aprender a aprender», a hacer que el aprendizaje sea verdaderamente significativo y a propiciar la interacción activa de todos los participantes del proceso educativo (Jaramillo, 2012).

Como se puede observar, las redes telemáticas están ofreciendo muchas posibilidades a la educación, tanto para propiciar la interacción con otras personas como para intercambiar información. En el contexto educativo, principalmente, se ha fortalecido la interacción entre maestros y alumnos, pero también entre pares o iguales. Esto ha resultado mucho más fácil gracias al uso de plataformas, como las LMS (learning management system), que aún se continúan desarrollando; plataformas de paga, tales como WebCT, Blackboard o Lotus; plataformas de uso libre, como Moodle, Interact, Claroline, Fle3 o Manhattan Active; así como por el movimiento, que actualmente está de moda, de las weblogs (Cabero, 2006).

Una plataforma está formada por diversas aplicaciones informáticas que se encuentran instaladas en un servidor, el cual permite al profesor administrar, gestionar, crear y distribuir cursos en internet (Sánchez, 2009). Las plataformas educativas son un elemento importante para estudiar y explorar, puesto que, al investigar un entorno virtual de aprendizaje, podemos conocer el desarrollo, el movimiento y la interacción que se generan entre pares y con el profesor. Por esta razón, las instituciones educativas están haciendo su incursión en la educación virtual o en la educación a distancia, debido a que existe una gran motivación para hacer algo diferente, puesto que repetir un modelo de enseñanza tradicional ya no resulta ser lo más viable (Ramírez, 2007, citado en Gros, 2011):

Tanto desde el mundo científico como desde otros ámbitos como la divulgación o la ficción, imaginar el futuro ha sido una de las temáticas más recurrentes a lo largo del siglo XX y en la primera década del actual (p. 145). 
Sin duda, las tecnologías nos ofrecen desafíos, y es una realidad que los nuevos entornos digitales son más complejos, por lo que los docentes y las instituciones deben estar en constante renovación y capacitación, de forma que puedan atender a las exigencias de nuestra sociedad actual (Quirós, 2009).

A raíz de lo anterior, las instituciones educativas, principalmente aquellas que imparten educación superior, han transformado sus procesos de enseñanza y aprendizaje por medio de la utilización de diversas modalidades educativas; tal es el caso del b-learning (blended learning) (Hernández y Romero, 2011).

Por tanto, este trabajo tiene como objetivo conocer la importancia de los entornos virtuales en los procesos educativos en el nivel superior. $Y$ de manera complementaria presenta el concepto de "entornos virtuales", describe sus características, los principales usos dentro del contexto universitario, así como los beneficios y los obstáculos de su empleo en los procesos educativos, retomando las aportaciones de diversos autores que han desarrollado investigación empírica y teórica en torno a este tema.

\section{Objetivos}

\subsection{Objetivo general}

Conocer la importancia de los entornos virtuales en los procesos educativos en el nivel superior.

\subsection{Objetivos específicos}

Se resumen en los siguientes:

- Definir el concepto de «entornos virtuales» basándose en las aportaciones de diversos autores.

- Describir las principales características de los entornos virtuales en los procesos de aprendizaje.

- Describir los usos de los entornos virtuales en los procesos de aprendizaje.

- Describir los beneficios de los entornos virtuales en los procesos de aprendizaje.

- Describir los obstáculos de los entornos virtuales en los procesos de aprendizaje. 


\section{Justificación}

Actualmente, existen diversos cambios en las estructuras sociales, en la forma de comunicación y en el uso de nuevos recursos y herramientas digitales que han impactado de modo considerable en distintos ámbitos de la sociedad, principalmente, en el terreno educativo. En este sentido, la transformación de este campo ha propiciado una amplia variedad de recursos que brindan de forma directa nuevas posibilidades para hacer eficientes los procesos educativos; sin embargo, esto no es una tarea fácil, pues son muchas las dificultades que se presentan en la educación.

Al respecto, en el contexto de la educación actual, ya no es suficiente con una enseñanza centrada en la transmisión de conocimientos en un aula tradicional; es decir, este mecanismo derivado del siglo XX ya no resulta ser funcional para las nuevas necesidades y características de la sociedad del siglo XXI. Por lo tanto, se ha optado por desarrollar mecanismos que generen un espacio de interacción más allá de las aulas y que faciliten el proceso de enseñanza-aprendizaje de forma óptima.

Estos nuevos entornos de aprendizaje transfor-

En el contexto de la educación actual, ya no es suficiente con una enseñanza centrada en la transmisión de conocimientos en un aula tradicional; es decir, este mecanismo derivado del siglo XX ya no resulta ser funcional para las nuevas necesidades y características de la sociedad del siglo XXI man la idea clásica de una enseñanza tradicional y establecen la educación virtual como una opción innovadora que favorece un ambiente activo y colaborativo de aprendizaje. En consecuencia, esta nueva forma de ver la educación no se trata de una moda, ni de algo pasajero, sino que es una realidad que va adquiriendo mayor relevancia en la oferta académica de diversas instituciones educativas y que evoluciona a partir de las necesidades y demandas de los estudiantes, las cuales se pretenden satisfacer mediante la implementación de herramientas tecnológicas (Moreira-Segura et al., 2014).

Dadas las tendencias actuales en el ámbito de la educación, se debe fomentar un aprendizaje constante y a lo largo de la vida, así como la autonomía del estudiante. En este aspecto, las tendencias generadas, principalmente, en el ámbito de la educación superior se enfocan en cinco aspectos, según establecen Peláez, Montoya, Gaviria y Acevedo (2015): cobertura, calidad, sociedad del conocimiento, pertinencia y pedagogía y currículo. De este modo, se resalta que la cobertura es un punto importante que hay que considerar debido a que, en México, la demografía está en aumento constante, por lo que la cobertura educativa se debe garantizar a cada ciudadano. Adicionalmente, la sociedad del conocimiento también ofrece una oportunidad para incluir nuevas TIC en los contextos educativos actuales.

En este sentido, la presente investigación se centrará en analizar la importancia de los entornos virtuales en los procesos educativos, abordando su evolución, sus características 
y todos los factores que los conforman, mediante el análisis detallado de la evolución de los mismos, sus aplicaciones y sus usos dentro de la educación, y se establecerá su importancia en los procesos educativos, ya que la utilidad de este estudio radica en brindar un panorama general sobre el tema a las instituciones educativas, a los emprendedores de la educación y a todos los individuos que se encuentren interesados en trasladarse a entornos virtuales para recibir o proporcionar educación.

Uno de los motivos principales que fundamenta esta investigación es la importancia de los entornos virtuales para la educación, ya que la tecnología está presente en nuestra vida diaria, puesto que nos acompaña en la mayoría de las actividades que realizamos, y, por supuesto, la educación no es la excepción. A partir de ello, se pretende proporcionar una visión actual sobre el uso de los entornos virtuales en los procesos educativos y, con ello, reconocer su importancia en el nivel superior.

Este estudio pretende esclarecer algunos interrogantes en torno al tema y proporcionar una mayor información al respecto para convertirse en un punto de partida en esta toma de decisiones y en la mejora de las prácticas educativas, principalmente, en el ámbito universitario.

\section{Metodología}

En este trabajo se lleva a cabo una revisión sistemática y descriptiva de bibliografía (Delgado, 2010; Medina-López, Marín-García y Alfalla-Luque, 2010) y se sigue el proceso propuesto por Medina-López et al. (2010), que consiste en cinco etapas:

- Respecto a la primera etapa, denominada «Identificación del campo de estudio y del periodo de análisis documental», fueron objeto de estudio los artículos, las ponencias y las tesis de maestría y doctorado que abordaban aspectos relacionados con los entornos virtuales en los procesos educativos en el nivel superior. Concisamente, para la identificación de los documentos, fueron seleccionados trabajos con las siguientes temáticas:

- Entornos virtuales de aprendizaje en docentes o estudiantes universitarios.

- Entornos virtuales de enseñanza en educación superior o universitaria.

Los temas relacionados fueron ambientes virtuales, entornos virtuales de aprendizaje, plataformas educativas, educación virtual, educación a distancia y entornos virtuales educativos.

El periodo de análisis establecido ha comprendido desde 2011 a 2019, dado que se deseaba conocer la evolución y las connotaciones recientes asignadas a la 
temática en contextos de educación superior, así como los principales conceptos, características, usos, beneficios y obstáculos, con el objetivo de identificar tendencias en este contexto.

- Con relación a la segunda fase, "Selección de las fuentes de información», se seleccionaron en total 22 documentos, de los cuales 3 fueron tesis de maestría, 1 tesis doctoral, 1 ponencia y 17 artículos científicos provenientes de diversas revistas electrónicas sobre temas de educación, educación superior, tecnología, educación a distancia e innovación. Algunas revistas que se utilizaron fueron International Journal of Technology and Educational Innovation, Revista Colombiana de Educación, Killkana Sociales, Ciencias Holguín, Píxel Bit: Revista de Medios y Educación, Revista Iberoamericana de Educación y Revista Razón y Palabra, entre otras. Cada una de estas publicaciones presenta un proceso de evaluación por pares que otorga fiabilidad al proceso de evaluación seguido para determinar la calidad de los artículos. Asimismo, se resalta que la mayoría de los artículos corresponden a países de América Latina y a algunos países de Europa.

- En la tercera fase, correspondiente a la «Realización de la búsqueda», se llevó a cabo una búsqueda manual en bases de datos como Ebsco, Google Scholar, Dialnet y en repositorios de tesis de diversas universidades latinoamericanas y españolas (Universidad Nacional de Colombia, Universidad de la República [Uruguay], Universidad Norbert Wiener y Universidad de Granada) para identificar aquellas publicaciones que en el título o palabras clave tuvieran los siguientes términos: «entorno virtual de aprendizaje» o «entorno virtual», «estudiantes universitarios», «docencia universitaria», «universitarios», «educación superior» y «entornos virtuales de enseñanza» 0 «ambientes virtuales de aprendizaje».

- En la cuarta etapa, denominada «Gestión y depuración de los resultados de la búsqueda», el objetivo fue seleccionar únicamente los documentos que abordaran los aspectos clave determinados en el inicio de la investigación. Cada uno de los artículos seleccionados fue revisado en su estructura y en los objetivos de investigación propuestos por el equipo de investigación para dilucidar si contenían los elementos clave que se querían analizar.

- Finalmente, en la última etapa, correspondiente al «Análisis de los resultados», se diseñó una ficha de revisión de bibliografía que apoyara la revisión sistemática de los documentos seleccionados y que contuviera las variables clave del estudio:

- Título.

- Autores.

- País de origen.

- Metodología empleada.

- Características de los participantes. 
- Definición de «entorno virtual».

- Características de los entornos virtuales.

- Usos de los entornos virtuales.

- Beneficios del uso de los entornos virtuales.

- Obstáculos en el uso de los entornos virtuales.

- Enlace electrónico.

\section{Resultados}

\subsection{Definiciones de los «entornos virtuales»}

Respecto a la primera unidad de análisis, se encontró que los «entornos virtuales» son entendidos por diversos autores como un software o aplicación informática que es utilizada con fines pedagógicos (Blanco y Anta, 2016; Cedeño, 2019; Jaramillo, 2012; López, 2015; López y Ortiz, 2018; Nóbile y Luna, 2015; Murrieta, 2016; Rodríguez y Barragán, 2017).

Por otro lado, desde la perspectiva de otros autores, un «entorno virtual» se refiere a un espacio, medio o ambiente en la red, donde es posible establecer una comunicación e interacción entre los usuarios-alumnos y el docente. Del mismo modo, en dicho espacio se concentran los materiales didácticos y recursos disponibles para el desarrollo de los procesos educativos (Bühl, 2013; Cedeño, 2019; Hiraldo, 2013; López, Flores, Rodríguez y De la Torre, 2012; Medina, Vialart y Chacón, 2016; Morado, 2017; Rodríguez y Barragán, 2017).

En contraste con lo anterior, los «entornos virtuales» también son definidos como recursos que complementan la gestión y la labor docente, dado que mejoran los procesos de enseñanza-aprendizaje y establecen la generación de actividades formativas orientadas a la adquisición de nuevos conocimientos y a la apropiación de los contenidos (Bühl, 2013; Cando, Alcoser, Villa y Ramos, 2017; Cedeño, 2019; Hiraldo, 2013; López y Ortiz, 2018; Montagud y Gandía, 2014; Nóbile y Luna, 2015; Valencia, Huertas y Baracaldo, 2014).

Además de lo antes mencionado, el término «entorno virtual» también se encuentra vinculado con un entorno educativo que utiliza herramientas de la web para realizar actividades similares a las que acontecen en las aulas regulares, es decir,
Los «entornos virtuales» son definidos como recursos que complementan la gestión y la labor docente, dado que mejoran los procesos de enseñanza-aprendizaje y establecen la generación de actividades formativas orientadas a la adquisición de nuevos conocimientos y a la apropiación de los contenidos


permite el establecimiento de actividades y la organización de temáticas para suscitar el aprendizaje de los estudiantes, de manera tal que estos se transforman en objetos de aprendizaje (López y Ortiz, 2018; Medina et al., 2016; Montagud y Gandía, 2014; Valencia et al., 2014).

Finalmente, el concepto de «entorno virtual» también es identificado como un sitio que promueve el aprendizaje de diversos temas de forma continua y permite el acceso al conocimiento y a la información (Guaña, Llumiquinga y Ortiz, 2015; Pérez, Duque y López, 2017).

\subsection{Principales características de los entornos virtuales}

En lo correspondiente a las principales características de los entornos virtuales en los procesos educativos de la educación superior, se encontró, basándonos en la revisión documental, que las características se orientan de acuerdo a dos categorías:

- Características de la dinámica acontecida en el uso del entorno virtual.

- Características de los componentes de los entornos virtuales.

En la primera categoría, diversos autores reconocen cinco características indispensables que los entornos virtuales en la educación superior deben tener ordenadas de acuerdo a la prioridad asignada por los autores. Estas características son colaboración, interactividad, flexibilidad, estandarización y escalabilidad (Blanco y Anta, 2016; Cando et al., 2017; Cedeño, 2019; Díaz y Maldonado, 2011; Guaña et al., 2015; Hiraldo, 2013; López et al., 2012; Marzoa, 2016; Morado, 2017; Murrieta, 2016). A continuación, en el cuadro 1, se incluye la definición de cada característica.

\section{Cuadro 1. Características de los entornos virtuales en la educación superior}

Característica

Colaboración

Interactividad

\section{Definición}

«Acciones que propician actividades multilaterales que tienen como objetivo la superación académica en las instituciones involucradas» (Universidad Nacional Autónoma de México [UNAM], 2018, p. 97).
"Relación entre el usuario y la herramienta tecnológica, o el usuario y los contenidos, durante el desarrollo de actividades de aprendizaje» (UNAM, 2018, p. 235). 
Característica

Flexibilidad

Estandarización

Escalabilidad
Definición

«Permite la diversificación de estructuras, modos y formas de organizar el estudio, el conocimiento y las competencias» (Ortega y Moreno, 2013, p. 48).

«Simplificar la utilización de tecnologías existentes y nuevas, centrándose en los interfaces y la interoperabilidad, reduciendo costes y complejidad, abriendo mercados y promoviendo un acceso más amplio a productos y servicios... [...] ayudan a garantizar la seguridad y desarrollar la confianza y la protección de los consumidores, mientras respetan los intereses legítimos de todos los interesados» (Bryden, 2003, citado en Segura, Bellver y Bellver, 2007, p. 2).

"Capacidad de la plataforma de e-learning de funcionar igualmente con un número pequeño o grande de usuarios» (Belloch, s. f., p. 3).

Fuente: elaboración propia.

Adicionalmente, se reconocen otras características de los entornos virtuales en la educación superior. Entre estas se encuentran las siguientes: funcionalidad, usabilidad, ubicuidad, accesibilidad, comunicación, autoaprendizaje e interconexión (Blanco y Anta, 2016; Cando et al., 2017; Díaz y Maldonado, 2011; Guaña et al., 2015; Hiraldo, 2013; Jaramillo, 2012; López et al., 2012; Morado, 2017; Murrieta, 2016).

En relación con la segunda categoría, orientada a las características de los componentes de los entornos virtuales, estos, usualmente, suelen incluir, de acuerdo al orden de prioridad, materiales multimedia, acceso al correo electrónico, evaluaciones, foros, chats, cuestionarios (autoevaluaciones) y simulaciones (Blanco y Anta, 2016; Bühl, 2013; Díaz y Maldonado, 2011; Guaña et al., 2015; Hiraldo, 2013; López y Ortiz, 2018; López, 2015; López, Flores, Rodríguez y De la Torre, 2012; Montagud y Gandía, 2014; Morado, 2017; Nóbile y Luna, 2015; Medina, Vialart y Chacón, 2016; Pérez, Duque y López, 2017).

También se reconocen otros elementos característicos. Entre ellos se encuentran los siguientes: ejercicios (actividades de aprendizaje), blogs, RSS (really simple syndication) y videoconferencias (Jiménez y Ortiz, 2018; López et al., 2012).

\subsection{Usos de los entornos virtuales en los procesos educativos}

Esta unidad de análisis dejó patente ciertas coincidencias en la postura de diferentes autores respecto a los usos de los entornos virtuales de aprendizaje en la educación superior. Por ejemplo, se detectó que algunos autores coinciden en que los entornos virtuales son utilizados 
principalmente para mejorar y explotar el proceso de enseñanza-aprendizaje, aumentando la calidad por medio del uso de las herramientas que contienen y que se brindan como apoyo didáctico (Cando etal., 2017; Cedeño, 2019; Díaz y Maldonado, 2011; Guaña et al., 2015; Hiraldo, 2013; Jaramillo, 2012; López, 2015; López et al., 2012; Medina et al., 2016; Montagud y Gandía, 2014; Murrieta, 2016; Nóbile y Luna, 2015; Valencia et al., 2014).

Por otra parte, otro punto importante dentro de este análisis es que los entornos virtuales de aprendizaje se usan para promover y propiciar el desarrollo de habilidades interpersonales por medio de la interactividad entre estudiantes y docentes, obteniendo como resultado un trabajo colaborativo por parte de todos los participantes, todo esto a través de dinámicas de grupo y trabajo cooperativo (Blanco y Anta, 2016; Bühl, 2013; Díaz y Maldonado, 2011; Marzoa, 2016; Medina et al., 2016; Rodríguez y Barragán, 2017).

De esta manera, la importancia de los entornos virtuales en la educación superior recae en el uso de estos como medio para complementar la educación presencial, obteniendo información, distribuyéndola, generando materiales educativos en formato digital (textos, imágenes, audio, simulaciones, juegos, etc.) y accediendo a ellos en el momento en el que se necesite para diferentes fines, como, por ejemplo, para la realización de debates y discusiones en línea acerca de algún tema (Blanco y Anta, 2016; Bühl, 2013; Cedeño, 2019; Hiraldo, 2013; López y Ortiz, 2018; Nóbile y Luna, 2015).

Finalmente, los autores estudiados consideran que uno de los usos más importantes de los entornos virtuales es la evaluación como medio de seguimiento del aprendizaje en los procesos educativos. En este caso, se menciona la importancia no solo de evaluar, sino también de proporcionar a los estudiantes una autoevaluación que les otorgue una retroalimentación (López 2015; López y Ortiz, 2018; Marzoa, 2016; Medina et al., 2016; Montagud y Gandía, 2014).

\subsection{Beneficios del uso de los entornos virtuales en los procesos educativos}

En esta unidad de análisis se presentan los beneficios del uso de los entornos virtuales en los procesos educativos. Conforme a lo anterior, se encontró que uno de los principales beneficios de dicho uso es la obtención de una mejor calidad educativa. El uso de estos entornos virtuales se caracteriza por brindar independencia para el aprendizaje, flexibilidad y disponibilidad en cuanto a tiempo y espacios disponibles, accesibilidad y rapidez en la 
obtención de materiales e información (Blanco y Anta, 2016; Cando et al., 2017; Cedeño, 2019; Medina et al., 2016; Montagud y Gandía, 2014).

Cabe señalar que diversos autores mencionan como beneficio destacable la mejora significativa del aprendizaje por medio de la motivación que surge al utilizar entornos virtuales, ya que, al hacerlo, se produce un mayor desarrollo de las habilidades de autorregulación de los estudiantes, así como de las cognitivas y creativas (Cedeño, 2019; Marzoa, 2016; López 2015; Medina et al., 2016; Montagud y Gandía, 2014; Valencia et al., 2014).

Aparte de esto, los autores consultados afirman que otro de los beneficios de usar entornos virtuales es que estos son un buen complemento para la educación presencial, ya que elevan la calidad educativa y disponen de múltiples herramientas que posibilitan el desarrollo de habilidades para el rendimiento académico de los estudiantes (Cando et al. , 2017; Cedeño, 2019; Hiraldo, 2013; Jaramillo, 2012; López, 2015; López et al., 2012; Marzoa, 2016; Medina et al., 2016; Montagud y Gandía, 2014; Valencia et al., 2014).

Por otra parte, tenemos como resultado que la comunicación y el trabajo colaborativo son factores que resultan ser beneficiosos para los usuarios de los entornos virtuales en la educación. Los estudiantes interactúan constantemente con otros estudiantes y con los docentes, propiciando relaciones interpersonales en el momento en que intercambian opiniones, trabajos e información académica (Blanco y Anta, 2016; Bühl, 2013; Hiraldo, 2013; Jaramillo, 2012; López, 2015; López y Ortiz, 2018; Díaz y Maldonado, 2011; Medina et al., 2016; Morado, 2017; Murrieta, 2016; Nóbile y Luna, 2015; Rodríguez y Barragán, 2017).

Por último, como mencionamos con anterioridad, los autores coinciden también en que un beneficio más en su uso es que los alumnos aprenden a evaluarse, a coevaluarse y a ser evaluados por sus docentes con el objetivo de dar seguimiento al aprendizaje obtenido (López, 2015; López y Ortiz, 2018; Marzoa, 2016; Montagud y Gandía, 2014).

\subsection{Obstáculos del uso de los entornos virtuales en los procesos educativos}

En este apartado, correspondiente a los obstáculos del uso de los entornos virtuales en los procesos educativos, se detectó que estos se organizan de acuerdo a los agentes implicados, es decir, en relación con las situaciones que presentan los docentes, los alumnos y la institución en general. En este sentido, en el siguiente cuadro se pueden ver los obstáculos encontrados desde la perspectiva de diversos autores. 


\section{Cuadro 2. Obstáculos del uso de los entornos virtuales en la educación superior}

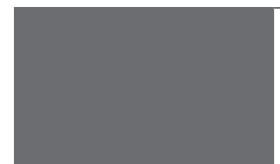

Temor o fobia respecto al uso de la tecnología y falta de información y conocimiento de metodologías y diseño de los cursos (Jaramillo, 2012; Rodríguez y Barragán, 2017).

Profesores

Dificultad para superar los paradigmas tradicionales de enseñanza (Valencia et al., 2014).

Necesidad o poco interés del profesorado en la formación del uso de los entornos virtuales (Blanco y Anta, 2016; Bühl, 2013; Guaña et al., 2015; Hiraldo, 2013, López, 2015; Morado, 2017).

No todos logran completar las actividades programadas en el entorno virtual (Medina et al., 2016).

Escaso uso de las herramientas de productividad propuestas en el entorno virtual (Díaz y Maldonado, 2011).

Alumnos

Insuficiencias en el aprendizaje de los alumnos y poca atención a los contenidos (Guaña et al., 2015).

Baja motivación para participar en los entornos virtuales (Blanco y Anta, 2016; Montagud y Gandía, 2014).

Poco dominio del uso del entorno virtual (Montagud y Gandía, 2014).

Dificultades en el límite de carga de archivos y accesos a las plataformas virtuales (Cando et al., 2017).

Uso de materiales complejos e incomprensibles para los usuarios (Montagud y Gandía, 2014).

Institucionales

Poca infraestructura tecnológica y diseño de apoyo tecnológico (López, 2015; López et al., 2012).

Dificultades derivadas del funcionamiento de la comunicación, lentitud en las transmisiones y en la red, averías en los servidores y poco reconocimiento del potencial de las tecnologías (López y Ortiz, 2018).

Fuente: elaboración propia.

A partir de los resultados encontrados, se reconoce que los agentes implicados, tanto docentes como alumnos, se enfrentan con diversos obstáculos que impiden el uso de los entornos virtuales en la educación superior y dificultan su interés por los mismos. Desde el punto de vista de estos autores, también existen algunos impedimentos derivados de aspectos institucionales, como la falta de infraestructura adecuada para lograr un óptimo funcionamiento en las instalaciones de la universidad.

\section{Docentes y alumnos se enfrentan con obstáculos que impiden el uso de los entornos virtuales en la educación superior, sin olvidar que también existen impedimentos institucionales, como la falta de infraestructura adecuada para lograr un óptimo funcionamiento}




\section{Discusión de los resultados}

Esta investigación tuvo como propósito conocer la importancia de los entornos virtuales en los procesos educativos en el nivel superior mediante el análisis del concepto de «entorno virtual» y las características más importantes que lo definen, así como los usos, beneficios y obstáculos que implica su inclusión en la educación.

En la actualidad, hacemos uso de las TIC en muchos aspectos de nuestra vida cotidiana, además de para nuestro aprendizaje. De esta manera, el concepto de «entorno virtual de aprendizaje» permite entender de qué manera usamos las TIC a la hora de aprender en la red de forma autónoma, haciendo así que no olvidemos la importancia que adquiere tener en cuenta el momento social y tecnológico en el que nos encontramos al reflexionar sobre los entornos de aprendizaje (Rodríguez y Castillo, 2019).

En este estudio se ha pretendido examinar la información existente respecto al concepto de «entorno virtual» basándose en diferentes autores, contrastando sus ideas y encontrando las similitudes o diferencias en relación a dicho concepto. A continuación, se discutirán los principales hallazgos de esta investigación.

De los resultados obtenidos en este trabajo se desprende que, actualmente, existe un conocimiento importante acerca de los entornos virtuales de aprendizaje, sus aplicaciones en la educación superior, sus características más importantes y los elementos o circunstancias que impiden o dificultan su inclusión en los procesos educativos.

En consecuencia, existen diferentes maneras de definir los «entornos virtuales de aprendizaje» y, basándose en los resultados de este estudio y en la literatura revisada, se presentan como espacios, softwares o aplicaciones informáticas que están dotados de materiales didácticos y recursos tecnológicos, donde la comunicación y la interacción son esenciales, ya que pretenden ser lo más parecido a un espacio real, un aula en la que se maneja la comunicación cara a cara, funcionando de esta manera en colaboración con los procesos pedagógicos y de aprendizaje.

Por otra parte, las características principales de estos entornos virtuales, y que han sido mencionadas por los autores estudiados, son colaboración, interactividad, flexibilidad, estandarización y escalabilidad, funcionalidad, usabilidad, ubicuidad, accesibilidad, comunicación, autoaprendizaje e interconexión. Asimismo, cuentan con componentes, tales como materiales multimedia, acceso al correo electrónico, evaluaciones, foros, chats, cuestionarios (autoevaluaciones) y simulaciones, ejercicios, blogs, RSS y videoconferencias.

Respecto al uso de los entornos virtuales de aprendizaje, los resultados muestran que son utilizados principalmente como medio de interacción en el que se comparte información a la que puede accederse en cualquier momento, con la finalidad de mejorar el aprendizaje $\mathrm{y}$, asimismo, tener la oportunidad de evaluar a los participantes. 
En cuanto a los beneficios de los entornos virtuales de aprendizaje, se relacionan con la mejora de la calidad educativa, ya que, además de complementar la educación presencial, se incrementa el aprendizaje y la motivación del estudiante, ya que este cuenta con mayor flexibilidad e independencia y más tiempo disponible para sus estudios; aprende a trabajar de manera colaborativa, manteniendo mayor comunicación con los demás participantes; y puede ser evaluado, evaluar y autoevaluarse.

Finalmente, se detectó que existen diversos obstáculos tanto para los alumnos como para los docentes y las instituciones. Estos obstáculos se vinculan con el temor que se genera ante la falta de conocimiento e información acerca del uso de estas herramientas tecnológicas. Además, otros obstáculos importantes que se detectaron es que no siempre se cuenta con la infraestructura requerida ni con el conocimiento respecto al complejo material que se necesita para implementar los ambientes virtuales de aprendizaje, sin olvidar la forma de resolver las dificultades técnicas o de diseño que pudieran surgir en el proceso de aprendizaje.

Una vez descrito todo lo anterior, los resultados de la investigación permiten responder al objetivo general de investigación acerca de la importancia de los entornos virtuales de aprendizaje en la educación superior.

\section{Conclusiones}

La educación está cambiando constantemente, por lo que es necesario encontrar herramientas útiles que produzcan cambios positivos en la comunidad y en sus problemas educativos. La educación virtual proporciona herramientas tecnológicas que sirven para resolver conflictos que existen en la educación presencial.

En este sentido, los entornos virtuales de enseñanza-aprendizaje surgen para ofrecer un espacio dentro de las crecientes redes informáticas y en la tecnología digital, produciendo ruptura de barreras geográficas, psicológicas y pedagógicas que adelantan procesos formativos en la educación superior (Guaña et al., 2015). Los entornos virtuales de aprendizaje se transforman en medios ideales para impartir una educación de calidad necesaria para los contextos del siglo XXI.

Centrándonos en los resultados de este estudio, se puede concluir que los entornos virtuales son espacios de comunicación o aplicaciones informáticas que proveen de oportunidades de aprendizaje ideales a los alumnos, quienes tienen la capacidad de interactuar con contenidos
Se puede concluir que los entornos virtuales son espacios de comunicación o aplicaciones informáticas que proveen de oportunidades de aprendizaje ideales a los alumnos, quienes tienen la capacidad de interactuar con contenidos y materiales adecuados para poder aprender 
y materiales adecuados para poder aprender. Por otro lado, los profesores tienen a su disposición un espacio para comunicarse, interactuar y enseñar de manera adecuada a los educandos por medio de mecanismos síncronos o asíncronos.

Asimismo, como principales características de los entornos virtuales de aprendizaje, se identificaron la colaboración, la interactividad, la flexibilidad, la estandarización y escalabilidad, además de la funcionalidad, la usabilidad, la ubicuidad, la accesibilidad, la comunicación, el autoaprendizaje y la interconexión. Asimismo, estos entornos virtuales cuentan con dos elementos: los recursos de aprendizaje y las herramientas de comunicación. Respecto a lo anterior, es importante considerar que, hoy en día, contar con un espacio abierto e interactivo que facilite el proceso de enseñanza-aprendizaje es una herramienta de utilidad, dado que se rompen las nociones de una educación tradicionalista y se promueve un aprendizaje autodirigido.

En relación a los usos de los entornos virtuales, la mayoría de los autores indican que son un medio de interacción entre los participantes (maestros-alumnos), además de que sirven para acceder a la información, compartirla e intercambiarla. También funcionan como herramienta de evaluación de los estudiantes.

Por otra parte, los beneficios de los entornos virtuales en los procesos de aprendizaje son variados; sin embargo, principalmente, se relacionan con la flexibilidad, la independencia y la disponibilidad de tiempo que representa su uso, por lo que indican que se puede percibir un incremento considerable en el aprendizaje. Unido a esto, también se fomenta en los alumnos la autonomía, el pensamiento crítico, el trabajo en equipo y la comunicación. Es posible que el participante realice ejercicios metacognitivos que le permitan conocer sus recursos y habilidades, y mejorar sus procesos de aprendizaje.

Para finalizar, en relación con los obstáculos de los entornos virtuales en los procesos de aprendizaje, en primera instancia se encuentra la resistencia al cambio por algunos profesores y la falta de conocimiento e información acerca del uso de estas herramientas tecnológicas por parte de los alumnos y de los docentes. Se consideró, además, como impedimentos importantes, la falta de equipos tecnológicos y de conocimiento técnico que se necesita para implementar los entornos virtuales de aprendizaje por parte de las instituciones educativas.

Los beneficios de los entornos
virtuales en los procesos de
aprendizaje son variados; sin
embargo, principalmente, se
relacionan con la flexibilidad, la
independencia y la disponibilidad
de tiempo que representa su
uso, por lo que indican que se
puede percibir un incremento
considerable en el aprendizaje.
Unido a esto, también se fomenta
en los alumnos la autonomía, el
pensamiento crítico, el trabajo
en equipo y la comunicación.
Es posible que el participante
realice ejercicios metacognitivos
que le permitan conocer sus
recursos y habilidades, y mejorar
sus procesos de aprendizaje

Los beneficios de los entornos virtuales en los procesos de aprendizaje son variados; sin embargo, principalmente, se relacionan con la flexibilidad, la independencia y la disponibilidad de tiempo que representa su uso, por lo que indican que se puede percibir un incremento considerable en el aprendizaje. en los alumnos la autonomía, el pensamiento crítico, el trabajo en equipo y la comunicación. Es posible que el participante realice ejercicios metacognitivos que le permitan conocer sus sus procesos de aprendizaje 


\section{Referencias bibliográficas}

Belloch, C. (s. f.). Entornos virtuales de aprendizaje, 1-9. Recuperado de <https://www. uv.es/bellochc/pedagogia/EVA3.pdf> (consultado el 21 de enero de 2020).

Blanco, A. y Anta, P. (2016). La perspectiva de estudiantes en línea sobre los entornos virtuales de aprendizaje en la educación superior. Innoeduca, 2(2), 109-116. Recuperado de <http://www.revistas.uma.es/index.php/ innoeduca/article/view/2032/1948> (consultado el 30 de enero de 2020).

Bühl, V. (2013). Los entornos virtuales de aprendizaje y sus usos en la enseñanza universitaria. Estado de situación y buenas prácticas en las Facultades de Química e Ingeniería de la Universidad de la República (Tesis de maestría). Universidad de la República, Uruguay.

Cabero, J. (2006). Comunidades virtuales para el aprendizaje. Su utilización en la enseñanza. Edutec: Revista Electrónica de Tecnología Educativa, 20, 1-20. Recuperado de $<$ www.edutec.es/revista/index.php/edu tec-e/article/viewFile/510/244> (consultado el 30 de enero de 2020).

Cando, A., Alcocer, F., Villa, H. y Ramos, R. (2017). Los entornos virtuales. Un plus en la docencia universitaria de la Escuela Superior Politécnica de Chimborazo. $3 \mathrm{C}$ Tic, Edición 22, 6(3), 26-42. Recuperado de <https://www.3ciencias.com/articulos/ articulo/los-entornos-virtuales-plus-la-do cencia-universitaria-la-escuela-superior-poli tecnica-chimborazo/> (consultado el 30 de enero de 2020).

Cedeño, E. (2019). Entornos virtuales de aprendizaje y su rol innovador en el proceso de enseñanza. ReHuSo: Revista de Ciencias Humanísticas y Sociales, 4(1), 119-127.

Delgado, M. (2010). Revisión sistemática de estudios: metaanálisis. Barcelona, España: Signo.
Díaz, V. y Maldonado, G. (2011). El alumnado universitario cordobés y la plataforma virtual Moodle. Píxel-Bit: Revista de Medios y Educación, 38, 121-128. Recuperado de <http://www.redalyc.org/articulo.oa?id= 36816200009> (consultado el 21 de enero de 2020).

Gros, B. (2011). Evolución y retos de la educación virtual. Barcelona, España: UOC.

Guaña, E., Llumiquinga, S. y Ortiz, K. (2015). Caracterización de entornos virtuales de enseñanza aprendizaje (EVEA) en la educación virtual. Ciencias Holguín, 4, 1-16. Recuperado de <http://www.redalyc.org/ar ticulo.oa?id=181542152006 $>$ (consultado el 3 de febrero de 2020).

Hernández, G. y Romero, V. (2011). El b-learning en contextos educativos universitarios: posibilidades de uso. En F. Díaz-Barriga, G. Hernández y M. Rigo (Eds.), Experiencias educativas con recursos digitales: prácticas de uso y diseño tecnopedagógico (pp. 95-120). México: UNAM.

Hiraldo, R. (2013). Uso de los entornos virtuales de aprendizaje en la educación a distancia. EDUTEC, 1-14. Recuperado de <https:// www.uned.ac.cr/academica/edutec/memo ria/ponencias/hiraldo_162.pdf> (consultado el 30 de enero de 2020).

Jaramillo, A. (2012). Ambientes virtuales en el proceso educativo: modos de asumir el entorno virtual (Tesis de maestría). Universidad Nacional de Colombia.

López, C. (2015). El desarrollo de competencias profesionales en los entornos virtuales de aprendizaje en ingenierías. El caso de la ingeniería en informática (Tesis doctoral). Universidad de Granada, España.

López, E. y Ortiz, M. (2018). Uso de entornos virtuales de aprendizaje para la mejora del rendimiento académico en estudiantes 
de quinto grado en la institución educativa Pozo Nutrias 2 (Tesis de maestría). Universidad Norbert Wiener, Perú.

López, M. ${ }^{a}$ C., Flores, K., Rodríguez, M. ${ }^{a}$ A. y De la Torre, E. (2012). Análisis de una experiencia de entornos virtuales de aprendizaje en educación superior: el programa de cursos en línea del centro universitario del sur de la Universidad de Guadalajara, México. Revista Iberoamericana de Educación, 60, 97-115. Recuperado de <https:// rieoei.org/historico/documentos/rie60a06. pdf $>$ (consultado el 3 de febrero de 2020).

Marzoa, J. A. (2016). Efecto de un entorno virtual sobre el rendimiento escolar en un curso de Química en el CETMAR 07 Veracruz, Ver. Razón y Palabra, 20(2_93), 600-608. Recuperado de <https://www.revistarazonypalabra.org/index.php/ryp/article/view/30> (consultado el 8 de febrero de 2020).

Medina, I., Vialart, M. ${ }^{a}$ N. y Chacón, E. J. (2016). Los entornos virtuales de enseñanza aprendizaje en la asignatura Morfología Humana. Educación Media Superior: Sociedad, 30(3), 591-598. Recuperado de $<$ http://scielo.sld.cu/scielo.php?script=sci_ arttext\&pid=S0864-21412016000300012> (consultado el 10 de febrero de 2020).

Medina-López, C., Marín-García, J. A. y AlfallaLuque, R. (2010). Una propuesta metodológica para la realización de búsquedas sistemáticas de bibliografía. Working Papers on Operations Management, 1(2), 13-30. Recuperado de <https://polipapers.upv.es/index. php/WPOM/article/view/786> (consultado el 3 de febrero de 2020).

Montagud, M. y Gandía, J. (2014). Entorno virtual de aprendizaje y resultados académicos: evidencia empírica para la enseñanza de la Contabilidad de Gestión. Revista de Contabilidad, 17(2), 108-115. Recuperado de <https://www.sciencedirect.com/scien ce/article/pii/S1138489113000216> (consultado el 30 de enero de 2020).
Morado, M. ${ }^{a}$ F. (2017). Entornos virtuales de aprendizaje complejos e innovadores: una experiencia de creación participativa desde el paradigma emergente. Revista Electrónica Educare, 22(1), 1-17. Recuperado de $<$ https://dialnet.unirioja.es/servlet/articulo? codigo $=6234157>$ (consultado el 6 de febrero de 2020).

Moreira-Segura, C., Delgadillo-Espinoza, B., Sánchez-Calvo, L. y Alvarenga-Venutolo, S. (2014). La virtualidad en los procesos educativos: reflexiones teóricas sobre su implementación. Tecnología en Marcha, 28(1), 121-129. Recuperado de <https://dialnet. unirioja.es/descarga/articulo/5051536.pdf> (consultado el 30 de enero de 2020).

Murrieta, R. (2016). Entornos virtuales de aprendizaje en educación superior: una experiencia con el uso del Moodle. Investigação Qualitativa em Educação, 1, 1.262-1.264. Recuperado de <https://proceedings.ciaiq.org/ index.php/ciaiq2016/article/view/727> (consultado el 6 de febrero de 2020).

Nóbile, C. y Luna, Á. E.(2015). Los entornos virtuales de enseñanza y aprendizaje en la Universidad Nacional de La Plata. Una aproximación a los usos y opiniones de los estudiantes. INNOEDUCA, 1, 3-9. Recuperado de <https://dialnet.unirioja.es/servlet/ articulo?codigo $=5363142>$ (consultado el 12 de febrero de 2020).

Ortega, S. C. y Moreno, M. ${ }^{a}$ C. (2013). La flexibilidad didáctica en entornos virtuales de aprendizaje. RevistaVirtu@almente, 1(2), 45-59. Recuperado de <https://journal.uni versidadean.edu.co/index.php/vir/article/ view/1409> (consultado el 3 de febrero de 2020).

Peláez, L. E., Montoya, J., Gaviria, A. S. y Acevedo, W. J. (2015). Tendencias de la educación superior. Estudios Regionales, 97, 133-163. Recuperado de <https://www. google.com/url?sa=t\&rct=j\&q=\&esrc=s\& source $=$ web \&cd $=\& v e d=2 a h U K E w j 2-K q t$ 
OprqAhUKIxQKHc_oAq8QFjAAegQIBR AB\&url=https\%3A\%2F\%2Fdialnet.unirioja. es\%2Fdescarga\%2Farticulo\%2F5264097. pdf\&usg=AOvVaw2zTS3712U-K-sPpFEgH $z d X>$ (consultado el 12 de febrero de 2020).

Pérez, H. A., Duque, F. J. y López, F. V. (2017). Entornos virtuales educativos. Un recurso para el aprendizaje de la matemática. Revista Atlante: Cuadernos de Educación y Desarrollo. Recuperado de <http://www.eumed. net/rev/atlante/2017/01/matematica.html> (consultado el 30 de enero de 2020).

Quirós, E. (2009). Recursos didácticos digitales: medios innovadores para el trabajo colaborativo en línea. Revista Electrónic@ Educare, 13(2), 47-62. Recuperado de <https:// www.google.com/url?sa=t\&rct=j\&q=\&esrc= s\&source $=$ web\&cd $=\& c a d=r j a \& u a c t=8 \&$ ved =2ahUKEwiwkoqD1JrqAhWBD2MBHYTrC 8gQFjACegQIARAB\&url=https\%3A\%2F\%2 Fwww.redalyc.org\%2Farticulo.oa\%3Fid\%3 D194114401005\&usg=AOvVaw2Pdt7Cqty h8NadJVvzuiHy> (consultado el 2 de febrero de 2020).

Rodríguez, M. C. y Barragán, H. M. (2017). Entornos virtuales de aprendizaje como apoyo a la enseñanza presencial para potenciar el proceso educativo. Revista Killkana Sociales, 1(2), 7-14. Recuperado de <https://www.researchgate. net/publication/320643236_Entornos_virtua les_de_aprendizaje_como_apoyo_a_la_ense nanza_presencial_para_potenciar_el_pro ceso_educativo> (consultado el 10 de febrero de 2020).

Rodríguez, B. y Castillo, C. (2019). Entornos virtuales de aprendizaje: posibilidades y retos en el ámbito universitario. Cuenca, España: Ediciones de la Universidad de Castilla-La Mancha.

Sánchez, J. (2009). Plataformas de enseñanza virtual para entornos educativos. PíxelBit: Revista de Medios y Educación, 34, 217-233. Recuperado de <https://www.reda lyc.org/pdf/368/36812036015.pdf> (consultado el 30 de enero de 2020).

Segura, J.A., Bellver, A. J. y Bellver, C.B. (2007). Entornos virtuales de aprendizaje y estándares de e-learning, 1-24. Recuperado de $<$ https://pdfs.semanticscholar.org/b29b/1b 3e3cc69deafcb01315839a17eee2b966ca. pdf> (consultado el 3 de febrero de 2020).

UNAM. (2018). Glosario. Recuperado de <https:// www.planeacion.unam.mx/Agenda/2007/ pdf/30_Glosario.pdf> (consultado el 6 de febrero de 2020).

Valencia, N. G., Huertas, A. P. y Baracaldo, P. O. (2014). Los ambientes virtuales de aprendizaje: una revisión de publicaciones entre 2003 y 2013, desde la perspectiva de la pedagogía basada en la evidencia. Revista ColombianadeEducación, 66, 73-103. Recuperado de <http://www.scielo.org.co/pdf/ rcde/n66/n66a04.pdf> (consultado el 6 de febrero).

Vidal, A. A. y Camarena, B. O. (2014). Retos y posibilidades de los cursos en línea a partir de una experiencia concreta. Píxel-Bit: Revista de Medios y Educación, 44, 19-34. Recuperado de <http://www.redalyc.org/pdf/368/ 36829340002.pdf> (consultado el 12 de diciembre de 2020). 


\section{Magisterio de Educación Infantil}

Hoy en día los centros educativos tienen una imperiosa necesidad de disponer de personal especializado, capaz de hacer frente a las necesidades educativas de la etapa infantil, de acuerdo con los conocimientos ya logrados por las diversas ciencias que hoy se ocupan de los niños en los primeros años de su vida, así como de los logros relativos al desarrollo de la inteligencia, la emocionalidad y la formación de la personalidad temprana, resultantes de estudios recientes sobre el desenvolvimiento de la mente infantil.

\section{Magisterio de Educación Primaria}

Son objetivos de la educación primaria, entre otros: conocer y apreciar los valores y las normas de convivencia, aprender a obrar de acuerdo con ellas, prepararse para el ejercicio activo de la ciudadanía y respetar los derechos humanos, así como el pluralismo propio de una sociedad democrática. También, desarrollar hábitos de trabajo individual y de equipo, de esfuerzo y responsabilidad en el estudio, así como actividades de confianza en uno mismo, sentido crítico, iniciativa personal, curiosidad, interés y creatividad en el aprendizaje.

\section{Menciones en los grados de} Magisterio de Educación Infantil y de Magisterio de Educación

\section{Primaria}

\section{Mención en Lengua Inglesa}

Mención en Pedagogía Terapéutica

Mención en Audición y Lenguaje

Mención en Tecnología Educativa

Mención en Enseñanza de la Religión Católica

\section{Curso de adaptación al grado}

Este curso de adaptación al grado ofrece a los maestros diplomados en la Especialidad de Educación Infantil o Primaria la posibilidad de obtener formación en campos determinados dentro del ejercicio profesional docente en estas etapas, a través de las menciones cualificadoras mencionadas anteriormente.

El objetivo principal del plan de estudios de este curso de adaptación al grado es contribuir a la actualización de la formación de los maestros diplomados. La aplicación de las TIC a la educación y de líneas pedagógicas innovadoras, fruto de la investigación en educación, hacen necesaria la actualización de los conocimientos didácticos de los diplomados y la formación de los maestros en investigación e innovación.

Al finalizar el curso de adaptación se obtiene el título de grado en Magisterio de Educación Infantil o en Magisterio de Educación Primaria.

La docencia en la etapa de educación infantil o primaria es una profesión regulada. Los graduados en Magisterio de Educación Infantil o en Magisterio de Educación Primaria tienen como principal salida profesional el trabajo como profesores en estas etapas, tanto en centros públicos como concertados y privados.

Si bien otras salidas profesionales para estos títulos pueden ser:

- Participación en proyectos educativos de organismos e instituciones (centros culturales, museos, asociaciones, ONG, etc.).

- Centros de educación para adultos.

- Centros de ocio y tiempo libre.

- Participación en programas de extensión educativa (actividades extraescolares, actividades de apoyo, etc.).

- Diseño y elaboración de materiales didácticos.

- Participación en proyectos de atención a la infancia y familiar. 